\title{
Das Problem der Menschenrechte bei Kant
}

\begin{abstract}
Kant wird oft als einer derjenigen großen Philosophen angesehen, dessen Werk wesentlich zum jetzigen Verständnis der Menschenrechte und Menschenwürde beigetragen hat. Kant scheint, wenn man in seine Schriften schaut, jedoch keine Theorie der Menschenrechte im modernen Sinne gehabt zu haben. Bei näherem Hinsehen zeigt sich folgender Grund: Kant unterscheidet zwischen dem bloß privaten Recht, das dem positiven Recht untergeordnet ist, und dem öffentlichen Recht, das die begrifflichen Bedingungen einer jeden legitimen, legalen Ordnung darstellt. Der Inhalt des öffentlichen Rechts wird bei ihm weder direkt aus einer freistehenden Moraltheorie abgeleitet, noch aus vertraglichen Übereinkünften oder dem positiven Recht. Stattdessen soll es aus den Ermöglichungsbedingungen einer rechtmäßigen Verfassung expliziert werden, unter der allein Ansprüchen auf (ein) „Recht“ irgendeine verbindliche Autorität zukommt. Wenn man diesen Ansatz ernst nimmt, kann man kaum eine Lesart bei Kant finden, die sich mit der modernen Auffassung von Menschenrechten vereinbaren lässt. Warum aber denken dann manche, dass Kant etwas zum modernen Verständnis der Menschenrechte beizutragen hätte? So lauten denn die Leitfragen der Erörterung: Welche Auffassungen in Kants Werk kommen einem zeitgenössischen Verständnis von Menschenrechten am nächsten? Warum jedoch können diese menschrechtlich vielleicht ähnlich klingenden Auffassungen Kants den heutigen Befürwortern der Menschenrechte doch keine Quelle oder Stütze bieten?
\end{abstract}

\section{Einleitung}

Am 10. Dezember 1948 verkündeten die gerade erst gegründeten Vereinten Nationen die Allgemeine Erklärung der Menschenrechte (AEMR). Mit ihr trat der anspruchsvolle Versuch in die entscheidende Phase, die im 17. Jahrhundert in der Tradition des Naturrechts entworfene und im 18. Jahrhundert in der Virgina Bill of Rights von 1776 und der französischen Déclaration des droits de l'homme et du citoyen von 1789 erklärte Idee der Menschenrechte als Maßstab einer weltweit verbindlichen Moralordnung zu etablieren. Menschenrechte sind - so lässt sich heute feststellen - in der zweiten Hälfte des 20. Jahrhunderts, bei allen Einschränkungen, zu einem global wirksamen, normativen Maßstab der Menschheit geworden. Obwohl sie weiterhin in manchen Staaten $\mathrm{zu}$ manchen Zeiten missachtet und verletzt werden, sind fast alle Menschen und Staaten bereit, die Menschenrechte faktisch als gemeinsamen moralischen Standard zu akzeptieren, 
auch wenn das teilweise nur ein Lippenbekenntnis ist. Die meisten Staaten haben die AEMR anerkannt und auch die sich darauf stützenden, jedoch jetzt völkerrechtlich bindenden Verträge und Menschenrechtskonventionen unterschrieben und ratifiziert. Kaum eine Regierung heute leugnet die Idee der Menschenrechte ganz, allenfalls versuchen sie sie anders zu interpretieren. Zwar gibt es leider weiterhin Völkermord, Folter, Gräueltaten und andere Formen staatlicher Willkür, aber Menschenrechtsverletzungen und die Missachtung der Menschenwürde werden von der Völkergemeinschaft geächtet, angeprangert und sie sind größtenteils durch die internationale Staatengemeinschaft rechtlich und politisch sanktionierbar. Ihre Sanktionierung beginnt sogar so langsam juristische und politische Realität anzunehmen. So beklagenswert die Verletzungen der Menschenrechte sind, bleibt doch festzuhalten, dass mit den Menschenrechten etwas erreicht ist, was in der bisherigen Geschichte ohne Beispiel ist: eine globale, transkulturelle und transnationale moralische Ordnung.

Unter „Menschenrechten“ versteht man dabei heute zumeist vorstaatliche individuelle Rechtsansprüche adressiert primär an die staatliche Ordnung. Dieses heutige Verständnis der Menschenrechte umfasst zumindest - bei allen unterschiedlichen Auffassungen - erstens vorstaatliche, moralische Ansprüche, die Menschen als Menschen haben, die sich zweitens auf grobes moralisches Unrecht beziehen, die es drittens zwingend erforderlich machen, dass es eine politische Ordnung gibt, die dieses grobe moralische Unrecht effektiv verhindert. Mit Menschenrechten ist also die an alle gerichtete Forderung verbunden, das jeweilige moralische Recht als positives oder legales Recht rechtstaatlich zu institutionalisieren, so dass Verletzungen dieses Menschenrechts mit staatlichen Zwangsmitteln sanktioniert werden können. Menschenrechte haben also eine Komponente eingebaut, die uns moralisch verpflichtet, sie auch rechtlich zu konkretisieren und $\mathrm{zu}$ institutionalisieren. ${ }^{1}$

Professionelle Philosophen könnten zur Selbstgratulation des eigenen Faches neigen, sieht die Entwicklung der Menschenrechte bzw. ihrer Idee doch nach einer welthistorischen Erfolgsgeschichte des Fortschreitens der Vernunft und ihrer Realisierung aus. Was aber - so muss man sich - durchaus selbstkritisch - philosophisch-reflexiv fragen - hat den außerordentlichen Erfolg der Menschenrechte wirklich ermöglicht, und zwar gerade im Unterschied zu sonstigen Normen der Moral, der Idee liberaler Grundrechte und demokratischer Politik? ${ }^{2}$

Waren es in der Tat unsere großen Philosophen und deren Ideen, die diesen Siegeszug ermöglichten? Im Folgenden möchte ich mich einem besonderen phi-

1 So schon Kant in RL, AA 06: 312.

2 Zu einem philosophischen Versuch einer Erklärung dieses Phänomens s. Gosepath 2009. 
losophiegeschichtlichen Fall in der Geschichte der Menschenrechtsidee zuwenden, nämlich Immanuel Kant, weil er immer wieder - meines Erachtens fälschlicherweise, wie ich im Folgenden zeigen möchte - als Mitbegründer der Idee der Menschenrechte angesehen wird.

Kant wird oft als einer derjenigen großen Philosophen angesehen, dessen Werk wesentlich zum jetzigen Verständnis der Menschenrechte und Menschenwürde beigetragen hat. ${ }^{3}$ Wenn man von heute, also aus der Perspektive der gegenwärtigen Politischen Philosophie der Menschenrechte auf Kant (zurück) schaut, ist mancher überrascht, nichts Vergleichbares in Kants Schriften zu finden. Wenn man sich nicht besonders gut in Kants Theorie auskennt, könnte man sehr wohl erstaunt sein, nirgends in seinen Schriften so etwas wie das, was wir heute „Menschenrechte“ nennen, entdecken zu können. ${ }^{4}$ Kant scheint, wenn man in seine Schriften schaut, offenbar keine Theorie der Menschenrechte im modernen Sinne gehabt zu haben. Bei näherem Hinsehen zeigt sich folgender Grund: Kant unterscheidet zwischen dem bloß privaten Recht, das dem positiven Recht untergeordnet ist, und dem öffentlichen Recht, das die begrifflichen Bedingungen einer jeden legitimen, legalen Ordnung darstellt. Der Inhalt des öffentlichen Rechts wird bei ihm weder direkt aus einer freistehenden Moraltheorie abgeleitet, noch aus vertraglichen Übereinkünften oder dem positiven Recht. Stattdessen soll es aus den Ermöglichungsbedingungen einer rechtmäßigen Verfassung expliziert werden, unter der allein Ansprüchen auf (ein) „Recht“ irgendeine verbindliche Autorität zukommt. Wenn man diesen Ansatz ernst nimmt, kann man kaum eine Lesart bei Kant finden, die sich mit der modernen Auffassung von Menschenrechten vereinbaren lässt.

3 So beispielsweise explizit bei Bielefeld 1998, 45 - 47, Höffe 1990, 135-145 und 2000, König 1994, 186.

4 Schon der Ausdruck „Menschenrecht(e)“ findet sich äußerst selten in Kants Schriften, schon gar kaum im Plural; hinzu kommen semantische Äquivalente wie „Rechte der Menschheit“. Für Belege der wenigen Stellen s. Höffe in diesem Band, Horn 2014, 75 - 84; Hruschka 2015, 1518f., zur Diskussion vgl. auch König 1994 und Müller 1999. In (fast) allen angeführten Stellen verwendet Kant „Menschenrecht(e)“ nicht im heutigen Sinn. Meist meint er damit die positiven Rechte bzw. die Rechtsordnung. So z. B. in der Fußnote zur ,Allgemeinen Anmerkung' (RL, AA 06: 320 -322). Im hier interessierenden naturrechtlichen Sinn subjektiver Rechte unabhängig vom positiven Recht spricht Kant vielleicht im Gemeinspruch von ihnen als den „heiligsten Menschenrechte[n]“ (TP, AA 08: 307). - Verschiedene Autoren benennen Beispiele für Menschenrechte bei Kant, etwa wenn Kant die Freiheit der Feder im Gemeinspruch (TP, AA 08: 304) als „einziges Palladium der Volksrechte“ bezeichnet oder wenn er in derselben Schrift sagt: „Ein jedes Glied des gemeinen Wesens hat gegen jedes andere Zwangsrechte“ (TP, AA 08: 291). Ich schließe mich Horns Interpretation $(2014,79-83)$ an, dass diese Beispiele nicht für Menschenrechte in unserem Sinne bei Kant stehen. 
Warum aber denken dann manche, dass Kant etwas zum modernen Verständnis der Menschenrechte beizutragen hätte? So wird denn die Leitfrage der folgenden Erörterung lauten: Welche Auffassungen in Kants Werk kommen einem zeitgenössischen Verständnis von Menschenrechten am nächsten? Warum jedoch können diese menschenrechtlich vielleicht ähnlich klingenden Auffassungen Kants den heutigen Befürwortern der Menschenrechte doch keine Quelle oder Stütze bieten?

Für die Zwecke meiner Erörterung werde ich Kants Unterscheidung zwischen drei Arten der Verpflichtungen (TP, AA 08: $277 \mathrm{f}$.) aufgreifen, von denen jede in einer anderen Sphäre gültig ist:

1. Erstens die Sphäre der Moralität überhaupt, wie sie Kant vor allem in der Grundlegung zur Metaphysik der Sitten entwickelt: Die Moral ergibt sich aus dem Standpunkt eines jeden moralisch urteilenden Individuums, von dem aus es seine Urteile darüber fällt, wie es sein eigenes Handeln ihm selbst, anderen Individuen und der Menschheit im Allgemeinen gegenüber rechtfertigen kann. Vom Standpunkt der Moral aus gesehen müssen wir - Kant zufolge - stets fragen: Was sollte ich als Individuum tun in meiner Beziehung $\mathrm{zu}$ anderen Individuen, denen gegenüber ich keine Verpflichtungen der politischen Gerechtigkeit habe? Die Bedingungen der Moral betreffen die Beziehungen zu mir selbst, zu anderen, mit denen ich nicht politisch verbunden bin (mit denen ich mich also im Naturzustand befinde), und $\mathrm{zu}$ anderen, denen gegenüber ich zwar politische Verpflichtungen habe, die aber nicht erschöpfend sind - zum Beispiel wenn ich bestimmten Personen gegenüber jenseits meiner positiv-rechtlichen Verpflichtungen auch moralische Verpflichtungen als Freund, Familienmitglied, Nachbar oder Geschäftspartner habe.

2. Zweitens die Sphäre der Politik oder des Staatsrechts, wie sie Kant vornehmlich in der Rechtslehre, dem ersten Teil der Metaphysik der Sitten ${ }^{5}$ bestimmt: Hier nimmt man den Standpunkt des Staates ein bzw. den der Staatsbürger(inn)en, die verpflichtet sind, die Gesetze ihrer Staaten zu achten als eine Weise ihren moralischen Verpflichtungen untereinander nachzukommen. Während die Forderungen der Moral auch im Naturzustand gelten (z.B. die Forderung, dass man niemanden zum eigenen Vorteil töten darf), gelten die Anforderungen des Rechts vollständig nur in der bürgerlichen Gesellschaft. In dieser gibt - Kant zufolge - jedes Individuum sein Recht auf, selbst zu bestimmen, wo die Grenzen zwischen der eigenen Freiheit und der

5 Zu den Metaphysischen Anfangsgründen der Rechtslehre (Rechtslehre) vgl. Höffe 1999; Ripstein 2009; Rosen 1993; Timmons 2000. 
Freiheit anderer zu ziehen ist, und erkennt stattdessen den Staat als legitimen öffentlichen Richter an. Vom Standpunkt der politischen Gerechtigkeit oder des Staatsrechts aus lautet die entscheidende praktische Frage also: Wozu darf ich berechtigterweise meine Mitbürger(inn)en im Medium des Rechts zwingen, und wozu sind sie in reziproker Weise berechtigt, mich zu zwingen?

3. Die dritte und letzte Sphäre der Verpflichtung ist die des Völkerrechts, oder das, was wir heutzutage die Sphäre der Gerechtigkeit jenseits der Staaten, bei Kant der internationalen Gerechtigkeit nennen könnten: Vom Standpunkt der internationalen Gerechtigkeit aus müssen wir fragen: Welchen Bedingungen innerhalb von Staaten sowie in den internationalen und transnationalen Beziehungen dürfen wir nicht widersprechen, und welche sind wir sogar verpflichtet, hervorzubringen? Im Ewigen Frieden ${ }^{6}$ und der Metaphysik der Sitten, in denen Kant diesen Teil seiner politischen Theorie ausführlich ausarbeitet, unterscheidet Kant drei Ebenen der Gerechtigkeit: Im Unterschied zum Staatsbürgerrecht, das innerhalb einzelner Staaten gilt, gelten das Völkerrecht sowie das Weltbürgerrecht für die internationale Sphäre der Gerechtigkeit. Die von Kant gezogene Differenz zwischen dem Völkerrecht (RL, AA 06: 334 -336) und dem Weltbürgerrecht (RL, AA 06: 352f.) soll dabei den Unterschied angemessen aufnehmen, dass eine gerechte Ordnung jenseits der Staaten nicht nur gerechte Beziehungen zwischen den Staaten, sondern auch zwischen den Staaten und der Gesamtheit der Individuen beinhaltet. Deshalb sollen - nach Kant - die Individuen jenseits ihres eigenen Staates den Status von Weltbürgern innehaben. Die drei Definitivartikel des Ewigen Friedens entsprechen diesen drei Ebenen der Gerechtigkeit: (1) „Die bürgerliche Verfassung in jedem Staate soll republikanisch sein“ (ZeF, AA 08: 349), (2) „Das Völkerrecht soll auf einen Föderalismus freier Staaten gegründet sein“ (ZeF, AA 08: 354), (3) „Das Weltbürgerrecht soll auf Bedingungen der allgemeinen Hospitalität eingeschränkt sein“ (ZeF, AA 08: 357).

Um etwas in Kants Werk zu finden, das dem modernen Verständnis von Menschenrechten nahekommt, schaue ich im Folgenden drei Kandidaten in Kants Theorie etwas näher an, denen man Ähnlichkeit zum heutigen Menschenrechtsverständnis unterstellen könnte und unterstellt hat: 1. Menschenwürde in der Sphäre der Moral, 2. das angeborene Recht im Staatsrecht und 3. das Weltbürgerrecht in der Sphäre der globalen Gerechtigkeit.

6 Zu Zum ewigen Frieden vgl. Habermas 1996; Höffe 2011; Lutz-Bachmann/Bohman 1996. 


\section{Menschenwürde in der Sphäre der Moral}

Allgemein geht man davon aus, dass Kant Menschenwürde als einen absoluten inneren Wert aller menschlichen Wesen konzipiert. ${ }^{7}$ Ebenso wird weithin angenommen, dass dieser Wert den Grund darstellt, dessentwegen wir andere zu achten haben.

Nun ist Moralität die Bedingung, unter der allein ein vernünftiges Wesen Zweck an sich selbst sein kann [...] Also ist die Sittlichkeit und Menschheit, insofern sie derselben fähig ist, dasjenige, was allein Würde hat. (GMS, AA 04: $434 \mathrm{f}$.)

Dieses Zitat scheint nahezulegen, dass Kant die Würde als Begründung der Moral und a fortiori der moralischen Rechte und Pflichten betrachtet.

Menschenwürde wird von Kant charakterisiert durch Attribute wie ,innerer Wert“ (z. B. GMS, AA 04: 434f.) und „unbedingt“. Diese Zuschreibungen sollen anscheinend ausdrücken, dass der Wert des Menschen von nichts anderem abhängt: Menschen haben einen Wert allein aufgrund ihrer Menschlichkeit. Von diesem Wert wird oft behauptet, dass es der normative Grund sei, aus dem wir Achtung vor dem Menschen haben sollten. Dieser Wert wird von vielen außerdem als „unvergleichlich“ bezeichnet, was bedeutet, dass er nicht gegen andere Werte abgewogen werden kann, etwa gegen den Wert von Dingen.

Das scheint einem heute weit verbreiteten Verständnis zu entsprechen. Einer solchen, prominenten heutigen Lesart zufolge ist Menschenwürde eine nicht-relationale Werteigenschaft menschlicher Wesen, die normative Forderungen generiert, Menschen und ihre Rechte zu achten. Dies ist nach verbreiteter Meinung das zeitgenössische Verständnis der Würde, das zum Beispiel der UN-Menschenrechtserklärung zugrunde liegt, in der von der „inhärenten Würde aller Mitglieder der Menschheitsfamilie“ die Rede ist, und der üblichen Auslegung von Art. 1 Abs. 1 des deutschen Grundgesetzes entspricht. Wenn Würde ein innerer Wert ist und die Menschheit Würde hat, dann hat auch die Menschheit einen inneren Wert, und von dort aus ist es nur ein kurzer Schritt zu der Konklusion, dass man die Menschheit aufgrund ihres inneren Werts zu achten hat.

Menschenrechte im modernen Sinn werden oft in derselben Weise aus der Würde des Menschen abgeleitet. Es wird dann behauptet, wir hätten Menschenrechte, weil wir Würde hätten. Und wir haben Würde - so lässt sich kantisch ergänzen - aufgrund unserer Fähigkeit zur Vernunft und Autonomie.

Da Kant Menschenwürde als Grundlage der Moral konzipiert und da Menschenrechte in der Menschenwürde begründet und von ihr ableitbar seien, fänden

7 So beispielsweise Paton 1947, 189; Schönecker/Wood 2003, 142. 
wir in Kants Werk - nach dieser Auffassung - die notwendigen Voraussetzungen für ein modernes Verständnis der Menschenrechte.

Wie steht es um den Wahrheitsgehalt dieser populären Inanspruchnahme von Kant? Schlecht, will mir scheinen: Denn erstens ist dies gar nicht das, was Kant tatsächlich sagt. Und zweitens ist dies auch nicht die angemessenste, systematische Begründung der Menschenrechte. Dazu im Einzelnen nun noch etwas genauer:

1. Wie Kant-Interpreten (besonders Sensen 2009 und 2011; Horn 2014) betonen, sprechen mindestens vier Argumente gegen diese Würde-Lesart von Kant: Erstens hängt für Kant das Gute vom Rechten ab (i); zweitens hat nicht die Menschheit, sondern nur die Moral einen inneren Wert (ii); drittens begreift Kant die Würde nicht als eine nicht-relationale Eigenschaft (iii) und schließlich impliziert Menschenwürde bei Kant keine Unverletzlichkeit der Person seitens des Staates.

i) Bezüglich des ersten Punkts macht Kant in der Grundlegung klar, dass das Gute vom Rechten abhängt.

Denn es hat nichts einen Werth als den, welchen ihm das Gesetz bestimmt. Die Gesetzgebung selbst aber, die allen Wert bestimmt, muss ebendarum eine Würde d.i. unbedingten, unvergleichbaren Wert haben, für welchen das Wort Achtung allein den geziemenden Ausdruck der Schätzung abgibt, die ein vernünftiges Wesen über sie anzustellen hat. Autonomie ist also der Grund der Würde der menschlichen und jeder vernünftigen Natur. (GMS, AA 04: 436)

Es gibt daher bei Kant keinen unabhängigen Wert, der die normative Forderung andere zu achten begründen könnte. Vielmehr stellt sich für Kant die Beziehung genau andersherum dar: Man soll andere achten, weil es durch den Kategorischen Imperativ in der Reich-der-Zwecke Formel so vorgeschrieben wird. Kant behauptet, dass die Forderung seine Maximen zu universalisieren „im Grunde einerlei“ sei mit der Forderung andere zu achten (GMS, AA 04: 437 f.). Denn die Forderung, seine Maximen zu universalisieren, verlangt, dass man all jene Maximen, die dem Willen der betroffenen Subjekte nicht entsprechen, verwerfen muss, denn damit erweisen sie sich eben als nicht verallgemeinerbar. Das aber besagt dasselbe wie die Zweck-an-sich Formel. So heißt es in der Kritik der praktischen Vernunft: Ein vernünftiges Wesen ist

[...] keiner Absicht zu unterwerfen, die nicht nach einem Gesetze, welches aus dem Willen des leidenden Subjects selbst entspringen könnte, möglich ist; also dieses niemals bloß als Mittel, sondern zugleich selbst als Zweck zu gebrauchen. (KpV, AA 05: 87.24-26).

ii) Es ist wegen des moralischen Gesetzes - und nicht wegen irgendeines inneren Wertes des Menschen - dass wir Menschen achten sollen. Diese In- 
terpretation wird belegt durch die Tatsache, dass Kant in der Grundlegung und anderswo einen absoluten inneren Wert (fast) ausschließlich der Moral und nicht den Menschen als solchen zuschreibt. Kant begründet die Forderung, andere $\mathrm{zu}$ achten, also weder in einem absoluten inneren Wert aller Menschen, noch behauptet er einen solchen Wert. Der Würdebegriff spielt in Kants praktischer Philosophie noch nicht mal eine begründende Rolle für die Pflichten (Klemme 2012, 45).

iii) Der „innere Wert“ ist lediglich ein anderer Ausdruck für dasjenige, was wir jenseits seiner Nützlichkeit schätzen sollen, jedenfalls insofern wir völlig vernunftgeleitet sind.

iv) Man sollte Kants Trennung zwischen Moral und Recht beachten. Es ist mehr als unklar, ob Kant an irgendeiner Stelle in seiner Rechtsphilosophie der Menschenwürde einen rechtebasierenden absoluten Status zuweist.

v) Pointierte Passagen über Kriminelle in der Rechtslehre (RL, AA 06: 329f.) legen zudem nahe, dass Kant zugesteht, dass Verbrecher ihre Würde verlieren können (Horn 2014, 73 und 99). Kants Behandlung der Beteiligungsrechte von Frauen, Schutzrechte von unehelichen Kindern, Versklavungsrechte für Angehörige fremder Ethnien, Strafgefangene, Menschen im Krieg lassen Zweifel aufkommen, ob er tatsächlich alle Menschen, denen er Würde zuspricht, als auch mit unveräußerlichen Menschenrechten ausgestattet ansieht (Horn 2014, 84-98). Vielmehr scheint die Würde des Staatsbürgers (statt des Menschen) die zentrale Grundlage individueller Rechtsansprüche bei Kant (Horn 2014, 110).

2. Zur Frage der systematischen Interpretation der Menschenrechte: Nicht nur bei Kant, sondern auch unter uns Zeitgenossen ist Menschenwürde ein vieldiskutierter Begriff, dessen inhaltliche und argumentative Rolle in einer Theorie der Menschenrechte und in der Rechtspraxis umstritten ist.

i) Es ist keineswegs klar, dass Menschen gewisse gemeinsame Eigenschaften haben - wie etwa die Fähigkeit zur Vernunft -, die ihnen Würde verleihen. Weder haben alle Menschen diese Eigenschaften, noch im gleichen Umfang.

ii) Es ist auch nicht offensichtlich, weshalb genau diese Eigenschaften notwendig sein sollten - im Sinne einer notwendigen Begründung -, um Menschenrechte zu haben.

iii) Um einen naturalistischen Fehlschluss zu vermeiden, dürfen diese Eigenschaften, über die Würde supervenieren soll, nicht rein biologistisch aufgefasst werden.

iv) Wenn wir davon ausgehen, dass Autonomie diejenige Eigenschaft darstellt, die Würde begründen kann und soll, dann stellt sich die weitere Frage nach der Extension der Würde: Warum sollten nur jene Menschen, 
die über diese Eigenschaft verfügen, als Subjekte und Objekte der Moral und der Menschenrechte angesehen werden? Warum begründet die Autonomie bei rationalen Wesen deren moralischen Status? Die Fähigkeit zum Vernunftgebrauch, so sahen wir gerade, gibt uns doch vielmehr die Möglichkeit das moralische Gesetz zu erkennen, das heißt den Kategorischen Imperativ, und die Pflichten, die sich aus seiner Anwendung ergeben. Warum gelten Rechte und Pflichten nur für Wesen, die im Prinzip in der Lage sind diese auch selbst zu erkennen und aus Vernunftgründen zu befolgen?

Als ein erstes Zwischenergebnis kann ich also festhalten, dass es ziemlich fragwürdig ist - in Kantischer als auch systematischer Hinsicht -, ob Menschenwürde moralisch verstanden ein Wert ist, der eine begründende Rolle innerhalb einer Menschenrechtskonzeption spielen kann. So wende ich mich denn Kants politischer Philosophie zu.

\section{Ein einziges angeborenes Recht}

Kants Rechtsphilosophie und politische Philosophie beginnt mit einer einfachen, aber eindrucksvollen Auffassung von Freiheit als Unabhängigkeit von den Entscheidungen anderer Personen. Der normative Ausgangspunkt von Kants Politischer Philosophie liegt in einem ,angeborenen Recht' (ius innatum), das zunächst wegen seines angeborenen, d.h. naturrechtlichen, Charakters seiner Gleichheit und Allgemeinheit ein besonders aussichtsreicher Kandidat für ein Kantisches Äquivalent heutiger Menschenrechte $\mathrm{zu}$ sein scheint.

\section{DAS ANGEBORENE RECHT IST NUR EIN EINZIGES}

Freiheit (Unabhängigkeit von eines anderen nötigender Willkür), sofern sie mit jedes Anderen Freiheit nach einem allgemeinen Gesetz zusammenstehen kann, ist dieses einzige, ursprüngliche, jedem Menschen, kraft seiner Menschheit, zustehende Recht. (RL, AA 06: $237 \mathrm{f}$.

Der potentielle Eingriff in die Freiheit einer anderen Person schafft eine Art von Abhängigkeit. Unabhängigkeit verlangt daher, dass eine Person nicht der Willkür einer anderen Person unterworfen ist. Unabhängigkeit ist das grundlegende Prinzip des Rechts. Dieses garantiert zugleich gleiche Freiheit, und verlangt daher, dass keine Person der Willkür einer anderen unterworfen ist. Gleiche Freiheit garantiert also den Status des Menschen als eigener Herr (sui iuris), und so das Recht eines jeden Menschen auf Unabhängigkeit. 
Personen genießen Rechte nach Kant nur in einer Rechtsgemeinschaft, nicht aber im Naturzustand. Kant akzeptiert keine moralischen, universellen, kategorischen und unveräußerlichen Individualrechte. ${ }^{8}$ Die einzige Art und Weise, in der eine Pluralität von Personen ihre Freiheit so genießen können, dass sie mit der Freiheit anderer vereinbar ist, besteht darin, sich mit den anderen zu vereinigen und in einen rechtlichen Zustand einzutreten. Als systematische Verwirklichung der Freiheit eines jeden, zusammen mit der eines jeden anderen, unter einem allgemeinen Gesetz, ist der rechtliche Zustand nicht lediglich das Recht eines jeden Menschen für sich allein, sondern das von allen zusammengenommen. Freiheit unter dem öffentlichen Gesetz ist das Recht der Menschen als solches.

Der wesentliche Punkt hier ist, dass in Kants Rechts- und politischer Sphäre nicht der unilaterale Wille, sondern der „vereinigte Willen des Volkes“ die Quelle der Normativität ist. Das heißt, dass die normative Kraft des Allgemeinen Prinzips des Rechts im Konsens und der Übereinkunft vernünftiger Akteure verortet ist, die einander im kooperativen Modus der Achtung begegnen und sich gegenseitig als Mitglieder des Reichs der Zwecke anerkennen.

Man könnte hierin eine bemerkenswerte Parallele zwischen Moral und Politik bei Kant erkennen - sofern man einer kohärentistischen Lektüre von Kants praktischer Philosophie zustimmt, also annimmt, dass die Politische Philosophie Kants von seiner Moralphilosophie abhängig ist. ${ }^{9}$ Allerdings ist der Gegenstand der Übereinkunft der vernünftigen Akteure in beiden Bereichen verschieden. In der Politik ist es das zwingende Recht. „Das Recht ist also der Inbegriff der Bedingungen, unter denen die Willkür des einen mit der Willkür des andern nach einem allgemeinen Gesetze der Freiheit zusammen vereinigt werden kann“ (RL, AA 06: 230). Erzwungen werden darf dazu, was nötig ist, um allen den gleichen Personen-Status, d.h. als Freier, zu sichern im Staat (RL, AA 06: 203-205). Das

8 Dies zeigt Horn 2014, 68-84 und in diesem Band. Mit diesem Nachweis unter anderem argumentiert Horn auf andere, ergänzende Weise als ich für die mit meiner Auffassung konforme These, dass es bei Kant keine Menschenrechte im Sinne moralischer Grundrechte gibt.

9 Bekanntlich stehen sich zwei grundsätzliche Lesarten von Kants praktischer Philosophie gegenüber. Die Vertreter einer Abhängigkeitsthese auf der einen Seite argumentieren für die Abhängigkeit des Rechts von Prinzipien der Moralphilosophie bei Kant. Diese Abhängigkeitsthese, nach der Recht sich bei Kant letztlich aus dem Kategorischen Imperativ ableiten lasse, vertreten vor allem B. Ludwig, W. Kersting, O. Höffe, P. Guyer und G. Seel. Die Vertreter einer Unabhängigkeits- oder Trennungsthese auf der anderen Seite sehen hingegen Kants Verständnis von Recht und Moral als voneinander unabhängige normative Phänomene. Die Vertreter der Unabhängigkeitsthese wie J. Ebbinghaus, G. Geismann, M. Willaschek, A. Ripstein und A. Wood unterscheiden sich allerdings teilweise stark in ihren Argumentationen. Für diese Zuordnung der Sekundärliteratur zum Verhältnis von Kants Moral- und Rechtsphilosophie vgl. Mosayebi 2013, 3-7, und Horn 2014, Kap 1.; dort finden sich auch die entsprechenden Literaturhinweise. 
unterstreicht die kategorische Differenz, die Kant zwischen einer moralischen Forderung zieht, die das individuelle Gewissen bestimmt, und einer Forderung der Gerechtigkeit, die Zwang durch juridische Gewalt erlaubt. Kant zufolge kann man letztere - Forderungen des Rechts - nicht direkt aus der ersteren - Forderungen der Moral - ableiten, ohne die Vermittlung durch eine legitime Autorität. Kant besteht auf der Notwendigkeit legitimer öffentlicher Autoritäten als logischer Bedingung jeder rechtmäßigen Ordnung.

Menschenrechte heute werden allgemein als vorstaatliche individuelle Rechtsansprüche primär an eine staatliche Ordnung verstanden. Der zentrale Einwand, der sich aus Kants politischer Philosophie gegen unser heutiges Verständnis der Menschenrechte entnehmen lässt, ist, dass alle individuellen vorstaatlichen Rechtsansprüche nur vorläufig moralische Geltung beanspruchen können. Während Kant wohlintendierte Bemühungen von Individuen, die eigene Auffassung des eigenen Rechts zu verteidigen, im Naturzustand durchaus rechtfertigt, stellt privates Recht ihm zufolge in der bürgerlichen Gesellschaft keine legitime Alternative zur Autorität öffentlicher Institutionen dar. Jene operieren in Vertretung des Gemeinwillens, sie interpretieren und schützen private Rechte, aber beschränken sie auch gemäß den Erfordernissen öffentlichen Rechts. Letztere Erfordernisse sind Freiheit, Gleichheit und Unabhängigkeit aller. Dies sind die Bedingungen, unter denen der Einzelne Gesetze als Ausdruck des Gemeinwillens aller und somit als gerecht anerkennen kann.

Wenn man über die Möglichkeit einer politisch-rechtlichen Begründung der Menschenrechte bei Kant nachdenkt, darf man also zum einen nicht außer Acht lassen, dass für Kant politisch-rechtliche Entscheidungen immer in einem bestimmten institutionellen Kontext des Staates stattfinden müssen und in komplexe Kooperationsbeziehungen eingebettet sind. Noch wichtiger ist, dass für Kant jeder Anspruch auf irgendein Menschenrecht der Autorität öffentlicher Institutionen überantwortet und überlassen werden muss, die die Rechtsansprüche im Namen des Gemeinwillens interpretieren und ihnen entsprechen, aber eben gegebenenfalls auch als nicht mit diesem Gemeinwillen vereinbar abweisen. Die tatsächliche Entscheidung öffentlicher Institutionen darüber, welche Rechtsansprüche und Gesetze mit dem Gemeinwillen des gesamten Volkes übereinstimmen und darum gerecht sind, ist die zentrale Forderung der Gerechtigkeit an Rechtsansprüche jeder Art.

Kants Theorie kennt also keine a priori gerechtfertigten, vorstaatlichen Menschenrechtsansprüche, unabhängig von den tatsächlichen Entscheidungen öffentlicher Institutionen. Denn nach Kant erfüllt nur öffentliches Recht die Bedingungen einer legitimen rechtlichen Ordnung. Der Inhalt des öffentlichen Rechts aber ist weder direkt aus irgendeiner freistehenden Moralphilosophie, noch aus Konventionen oder positivem Recht einfach abzuleiten. Stattdessen 
muss er aus und unter den Bedingungen einer rechtmäßigen Verfassung expliziert werden, unter der allein Ansprüchen auf (ein) „Recht“ irgendeine verbindliche Autorität zukommt. Statt direkt zu bestimmen, welches rechtmäßige oder legitime Rechtsansprüche sind, definiert Kant also vielmehr die Bedingungen, unter denen ein legitimer Richter existieren kann, der diese Entscheidung trifft (für eine bestimmte Gruppe, in Bezug auf die diese Entscheidungen des Richters dann verbindlich wirken).

Kants einziges ,angeborenes Recht‘ eignet sich also nicht als Grundlage unseres heutigen Menschenrechtsverständnisses, weil sich aus ihm gerade keine spezifischen prä-politischen konkreten (Menschen-)Rechte ableiten lassen. Das angeborene Recht ist also zu unbestimmt, formal und relational, um entsprechend dem heutigen Menschenrechtsverständnis als naturrechtliche, bestimmte, substantive Rechtsansprüche gegenüber anderen fungieren zu können (Flikschuh 2015). Das ,angeborene، Recht kann auch nicht bloß eine Art Meta-Recht darstellen, aus dem sich dann konkretere Menschenrechte entwickeln ließen. Es kann auch nicht nur als eine Menschenrechtsschranke fungieren, die mögliche konkrete Menschenrechtskonstruktionen begrenzt. Denn diese beiden letzten Möglichkeiten sind mit Kants Forderung, dass Recht(e) nur aus tatsächlichen Entscheidungen öffentlicher Institutionen generiert werden können, unvereinbar. Es ist nicht die Abstraktheit, Unbestimmtheit, Un-Interpretiertheit des ius innatum allein, die dieses als Menschenrecht oder Menschenrechtsbasis ungeeignet sein lässt. Vielmehr bedarf es nach Kants zentraler politisch-philosophischer Auffassung, um von Rechten im vollen Sinne sprechen zu können, des Übergangs vom unilateralen zum omnilateralen Willen. Deshalb müssen wir nach Kant den Naturzustand verlassen und gemeinsam mit den anderen in den Staatszustand eintreten. Das ,angeborene Recht' kann deshalb also kein Menschenrecht im heutigen Sinne sein, weil dessen Anspruch sich unilateral gegen andere Individuen und noch schlimmer, gegen andere Personen im vereinigten Staats- und damit Rechtzustand richten würde.

\section{Völkerrecht und Weltbürgerrecht}

Kants generelle Forderung, dass Beziehungen zwischen den Staaten dem Recht unterzuordnen seien, könnte einen zunächst zu der Annahme verführen, Kant müsse eigentlich irgendeine Form der Weltregierung befürworten. Im Naturzustand zwischen Individuen entscheidet jeder einzelne, was als ein legitimer Rechtsanspruch gilt, der anderen und sich selbst aufgezwungen werden kann. Aus den daraus resultierenden Kontroversen zwischen Individuen entsteht für Kant die Notwendigkeit, einen Staat einzurichten, der im Namen des Gemein- 
willens des ganzen Volkes Recht spricht und durchsetzt. Die einzelnen Staaten befinden sich aber nach Kant zunächst einmal auch in einer Art Naturzustand zueinander. Deshalb läge es nahe, nun einzig einen Weltstaat als Lösung dieser Naturzustandsproblematik zu fordern. Tatsächlich aber weist Kant die vernünftige Möglichkeit einer solchen Weltregierung explizit zurück. Aber immerhin fordert er im dritten Definitivartikel seines Entwurfs Zum ewigen Frieden für alle Menschen ein Weltbürgerrecht jenseits ihrer Staaten, denen sie angehören (Hoesch 2015). Damit gibt es bei Kant ein Recht, das allen Menschen qua Menschseins und nicht qua Staatsangehörigkeit zusteht. Im wesentlichen Unterschied zu unserem heutigen Verständnis der Menschenrechte wird in Kants Theorie die Reichweite des weltbürgerlichen Rechts (ius cosmopoliticum, RL, AA 06: 352) explizit beschränkt auf die „Bedingungen der allgemeinen Hospitalität“ (ZeF, AA 08: 357), also eines „Besuchsrecht[s], welches allen Menschen zusteht, sich zur Gesellschaft anzubieten“ (ZeF, AA 8: 357).

Das ist wohl kaum das, was gegenwärtige Völkerrechtler im Sinn haben, wenn sie behaupten, dass Staaten in unserem gegenwärtigen Völkerrecht inzwischen den Menschenrechten verpflichtet seien. Diese Tatsache, dass staatliche Souveränität heute im Völkerrecht an die Anerkennung und Garantie der Menschenrechte durch den betreffenden Staat gebunden ist, ist wirklich ein bemerkenswerter normativer weltgeschichtlicher Fortschritt, den Kant aber weder vorhergesehen noch vernunftrechtlich gefordert hat.

Man schaue sich dazu Kants Argument an: Das Hauptanliegen seiner Konzeption einer gerechten internationalen Ordnung liegt darin, Beherrschung auszuschließen, erstens im Verhältnis zwischen republikanischen Staaten und zweitens zwischen diesen und unabhängigen Bürgern in diesen Staaten. Kant zufolge ist die einzige wirklich rechtliche internationale Ordnung diejenige, die die drei Definitivartikel des Ewigen Friedens erfüllt: In dieser Ordnung ist jeder Staat republikanisch verfasst. Die souveränen Völker als Staaten stehen zueinander in einer Beziehung der freien Föderation und des gegenseitigen Schutzes statt der Hierarchie und Beherrschung. Im Verhältnis zwischen den Staaten und einzelnen Fremden steht Nichtstaatsbürgern ein Weltbürgerrecht $\mathrm{zu}$, allerdings stark eingeschränkt auf das Recht auf nicht feindliche Behandlung als Nicht-Bürger.

Die republikanische Verfasstheit von Staaten ist deshalb gefordert, weil nur sie garantiert, dass die Beziehungen zwischen Bürgern gerecht sind, d.h. vereinbar mit der Freiheit aller geregelt sind, so wie es das Prinzip des öffentlichen Rechts erfordert. Dieselbe Forderung gilt aber auch für das Verhältnis zwischen Staaten: Ungerechte Hierarchien in den zwischenstaatlichen Beziehungen sind zu vermeiden. Während Kant einräumt, dass dieses Erfordernis im Prinzip (in thesi) durchaus durch eine allgemeine Weltrepublik verwirklicht werden könnte, gibt er $\mathrm{zu}$ bedenken, dass die Welt realiter (in hypothesi) bereits in Staaten aufgeteilt ist 
und diese der Einrichtung einer Weltrepublik nicht zustimmen würden. Unter diesen Bedingungen bestehe die einzige Möglichkeit, ungerechte Beziehungen der Beherrschung zwischen Staaten zu vermeiden, in der freiwilligen Entscheidung aller Staaten, einer Föderation beizutreten, die jeden von ihnen vor äußerlichen Angriffen schützt und gleichzeitig garantiert, dass keiner der Mitgliedsstaaten in die Angelegenheiten der jeweils anderen interveniert (ZeF, AA 08: 357; vgl. ZeF, AA 08: 346; RL, AA 06: 351). Dieses Argument ist nicht lediglich prudentiell oder pragmatisch begründet, sondern auch historisch. Kant ist zwar nicht der Überzeugung, dass grundsätzlich irgendetwas falsch sei mit einer allgemeinen Weltrepublik (ZeF, AA 08: 357), sondern vielmehr, dass ein solcher Zustand im Ausgang von den gegenwärtigen Bedingungen einer Welt der unabhängigen Einzelstaaten anders als durch ungerechte und darum unerlaubte Akte der Eroberung nicht zu erreichen ist. In einem Zustand der völligen Anarchie zwischen Individuen - einem regelrechten Naturzustand - mag es gerechtfertigt sein, andere zum Eintritt in einen bürgerlichen Zustand zu zwingen. Aber sobald mehrere bürgerliche Staaten existieren, hat jeder von diesen einen prima facie legitimen Anspruch, dass kein anderer Staat (oder Individuum, oder Staatenbund) unilateral in seine Angelegenheiten intervenieren darf. Dies ist Kants entscheidendes Argument für die Konzeption einer zwischenstaatlichen statt wirklich globalen Gerechtigkeit.

Von dieser Warte aus können wir auch verstehen, warum Kant vermutlich nahezu jeden Anspruch auf „Menschenrechte“, wie wir sie heute üblicherweise verstehen, zurückweist bzw. zurückweisen würde. Denn einen solchen Anspruch zu legitimieren hieße, den souveränen Staaten das Recht abzusprechen, selbst darüber zu entscheiden, welche Rechte ihre Bürger haben sollen und wie diese durchzusetzen sind. Kein Staat und keine Gruppe von Staaten kann für einen anderen Staat, sofern er republikanisch verfasst ist, im Namen der Gerechtigkeit entscheiden, welche Rechte dieser seinen Bürgern zu gewähren hat, denn dies wäre für Kant ein Fall von Ungerechtigkeit. Jedoch - so könnte man an dieser Stelle Kantianisch weiterdenken - könnten souveräne republikanische Staaten im Frieden miteinander ohne eine höhere supranationale Autorität sich selbst frei, gleich, reziprok, omnilateral an Menschenrechte binden. Aber - das ist für die Kantische Position im Ewigen Frieden von zentraler Bedeutung - dürfen sie dazu niemals gegen ihren souveränen Willen gezwungen werden (wie das im heutigen Völkerrecht aber der Fall sein könnte).

Man könnte nun jedoch annehmen, dass Kant die Möglichkeit von so etwas wie „Menschenrechten“ auf einer anderen Ebene einräumt.

(i) Ließen sich Menschenrechte mit Kant nicht doch als Teil eines Weltbürgerrechts denken? Könnten Menschenrechte nicht Teil eines übergeordneten Weltbürgerrechts sein, das der Souveränität von Einzelstaaten legitime Grenzen 
auferlegt? In der Tat sieht Kant, wie bereits erwähnt, zwar als rechtsphilosophische Innovation ein Weltbürgerrecht vor, aber lediglich eingeschränkt auf das Recht auf Hospitalität, was so viel bedeutet wie „nicht-feindselig behandelt zu werden“ (ZeF, AA 08: 358), um zu erlauben, in Kommunikation miteinander treten zu können (Eberl/Niesen 2011, 251). Die Tatsache, dass dieses so sehr abweicht von den üblichen Kandidaten auf bekannten Listen von Menschenrechten, ist vielsagend: Für Kant ist nur dieses eine Recht als ein weltbürgerliches vorstellbar, weil es aus keinerlei Prinzipien der Moral, der Metaphysik oder der menschlichen Natur abgeleitet ist - die ihrerseits freilich im Zweifelsfall durch die souveräne Entscheidung der Einzelstaaten aufgehoben würden. Kants Argument für das Recht auf Hospitalität ist weder unmittelbar moralisch noch politisch: Der Grund, aus dem es verboten ist, dieses Recht zu missachten, ist vielmehr der, dass diese Missachtung unserer Pflicht zuwiderlaufen würde, die Bedingungen zu achten und $\mathrm{zu}$ erhalten, unter denen individueller Zugang $\mathrm{zu}$ einer gerechten globalen Ordnung allein möglich ist. Vermutlich ist dieses Recht nicht gegen den souveränen Willen von Staaten durchsetzbar. In jedem Fall aber ist es kein unmittelbares moralisches Recht, das mit dem Recht der Staaten auf Souveränität konkurrieren würde, oder ein Gebot der Gerechtigkeit, das irgendjemand anderes als die Staaten selbst interpretieren und anwenden dürfte. Es entspricht also keineswegs dem herkömmlichen neuzeitlichen Verständnis der Menschenrechte. Das aus heutiger Perspektive überraschende Moment in Kants Argumentation ist also, dass er abgesehen von dem Recht auf Hospitalität jedes mögliche weitergehende Menschenrecht ausschließt, das die souveräne Unabhängigkeit republikanischer Staaten, wie sie im ersten und zweiten Definitivartikel des Ewigen Friedens definiert ist, beschränken könnte. Wenn man diesen Zusammenhang bei Kant erkennt, dann muss man auch einsehen, dass vieles von dem, was Vertreter von Menschenrechten heute propagieren, den Bedingungen der Möglichkeit von internationaler Gerechtigkeit aus Kantischer Sicht widerspricht und sie also wiederum aus Kants Sicht - sogar dieselben Werte untergraben, die sie zu befördern behaupten.

(ii) Vielleicht gibt es noch eine weitere Möglichkeit, Menschenrechte bei Kant zu verorten. Könnte man mit Kant „Menschenrechte“ als bindende Bedingungen für staatliche Legitimität denken? Menschenrechte könnten Minimalbedingungen darstellen, welche legitime Staaten erfüllen müssen, um überhaupt als legitime souveräne Staaten anerkannt werden zu können. ${ }^{10}$ Menschenrechte könnten dann als konstitutive Rechte verstanden werden, die jedes Gesetz beachten muss, das den Anspruch erhebt, dem Gemeinwillen des Volkes zugeschrieben werden zu

10 S. z.B. Stilz 2009 und Valentini 2012. 
können. Kant lehnt diese Möglichkeit indes ziemlich deutlich ab: Denn auch wenn es in gerechten Staaten immer möglich sein muss, eigene Ansichten in Fragen der Gerechtigkeit öffentlich zu äußern, so liegt der Zweck des Staates für Kant doch letztlich gerade darin, dass dieser als Richter in den öffentlichen Debatten um Gerechtigkeit auftritt, dessen Autorität nicht durch private Parteien oder andere Staaten oder Staatenbünde in Frage gestellt werden darf. Dies ist Kants berühmtberüchtigtes kategorisches Argument gegen ein Recht auf Widerstand/Rebellion/ Revolution (vgl. z. B. TP, AA 08: 303f.; RL, AA 06: 318-322). ${ }^{11}$ Falls man nun für das Recht von Individuen oder anderen Staaten argumentieren wollte, über andere Staaten zu urteilen und verbindlich für diese zu entscheiden (statt ihnen z. B. nur beratend die eigene Ansicht darzustellen), so stellte man damit gemäß Kants vertragstheoretischer Konzeption nun die Legitimität von Staaten generell in Frage. So dürften beispielsweise auch internationale Gerichtshöfe Staaten nicht menschenrechtlich verurteilen, denn das würde ihre Souveränität einschränken (Sangiovanni 2015, 677). Kant könnte diese einheitliche absolute Konzeption der Souveränität auch nicht ohne weiteres aufgeben, denn sie folgt aus seiner Theorie der notwendigen omnilateralen Zustimmung des ganzen Volkes für volle Rechtsgeltung. Nur der vereinigte Willen hat die Autorität Recht zu setzen. Jeder einzelne, der widerspräche, hätte nur einen unilateralen Willen. Aus diesem Argument Kants ergibt sich Kants strikte Zurückweisung eines Rechts auf Revolution. Wenn es auf einer höheren Ebene kein autorisiertes Organ gibt, dass den Disput zwischen dem Staat und den (aus moralischen Gründen) Rebellierenden lösen kann, dann läuft das nach Kant im Endeffekt darauf hinaus, dass die Rebellierenden eine Rückkehr in den Naturzustand verlangen. Das aber ist nach Kant „das höchste und strafbarste Verbrechen“ (TP, AA 08: 299), weil sich so die Bedingungen für Recht (s. RL, AA 06: 256) überhaupt auflösen. ${ }^{12}$

11 Zu Revolution bei Kant s. Flikschuh 2008; Guyer 2006, 284-294; Ripstein 2009, 325-352. 12 Ripstein (2009) diskutiert die Möglichkeit, ob Kant nicht wenigstens zulassen könne, dass gegen den Staat rebelliert wird, wenn dieser nicht einmal die Bedingungen des Rechtsstaats (rule of law) garantiere und damit barbarisch würde. Vgl. Byrd/Hruschka 2010, 91 und 181. Wie Sangiovanni (2015, 681-684) richtig und naheliegenderweise bemerkt, erlaubt die Bedingung der Rechtsstaatlichkeit zwar einige Unrechtsregime zu kritisieren (z. B. Nazi-Deutschland), aber diese Bedingung ist viel zu schwach, um auch nur etwas Vergleichbares annähernd den heutigen Menschenrechten zu generieren. 


\section{Kantische Auffassung von Menschenrechten}

Wenn es also keine Menschenrechtskonzeption bei Kant selbst gibt, dann kann man dennoch eine Auffassung vom Inhalt und der Begründung von Menschenrechten im Kantischen Geiste entwickeln. Dazu bezieht man sich auf einige Teile der Theorie Kants und vernachlässigt bewusst andere Teile in seiner Theorie, die der eigenen Auffassung entgegenstehen, oder widerspricht ihnen sogar mit guten Gründen. Solche Ansätze kann man dann kantische (mit einem kleinen „k“) bzw. Kantianische Menschenrechtstheorien nennen. Zwei naheliegende und nicht unplausible Möglichkeiten will ich abschließend noch erwähnen. ${ }^{13}$

a) Eine mögliche kantisch (mit kleinem „k“) inspirierte Menschenrechtstheorie nimmt ihren Ausgangspunkt bei Kants Zweck-an-sich Formel und Reichder-Zwecke Formel des Kategorischen Imperativs in der Grundlegung. Kant habe damit das zentrale Anliegen eines unverletzlichen Status von Personen als Personen auf den Begriff der Menschenwürde gebracht. Mit der Zweck-an-sich und der Reich-der-Zwecke Formel des Kategorischen Imperativs wird nach dieser verbreiteten Auffassung ein naturrechtlicher, universaler, absoluter und unveräußerlicher Status von Personen etabliert. Jede Rechtsordnung hat dementsprechend die Würde des Menschen zu achten und zu schützen. Das ist die Verpflichtung aller staatlichen Gewalt und Rechte. Die moralisch vorstaatlich, naturrechtlich begründete Würde des Menschen ist danach das tragende Konstitutionsprinzip und Ziel einer jeden Verfassung. In dieser Form muss Menschenwürde als oberstes Konstitutionsprinzip eine strukturbildende Funktion für

13 Nicht vielversprechend scheint mir hingegen der Versuch, Kants Moralphilosophie auf den Bereich der Politik direkt zu übertragen. Damit würde die moralphilosophische Prozedur Kants, der Kategorische Imperativ, direkt auf das die äußeren Freiheiten regelnde Recht übertragen. Unabhängig von der Debatte zwischen den Vertretern der Abhängigkeitsthese und der Unabhängigkeitsthese muss man wohl festhalten, dass Kant selbst eine solch direkte Übertragung der Moral auf das Recht so nicht vorgesehen hat. Aus guten Gründen, will mir scheinen. Ich erwähne nur einige: Moral kann in einem Rechtssystem kaum unmittelbar gelten. Das würde die Unterscheidung Kants von Moralität und Legalität einziehen. So müsste zunächst von diesem Ansatz gezeigt werden, warum moralische Pflichten legale Pflichten und Rechte generieren sollen. Manche moralische Pflichten gelten aus gutem Grund in unseren Rechtssystemen nicht, z. B. das Lügenverbot. In diesem Fall ist etwas moralisch verboten, was aber rechtlich erlaubt bleiben soll. Es müsste ferner erörtert werden, ob nach diesem Ansatz contra Kant auch für das Recht gelten soll, dass es aus der rechten Gesinnung heraus befolgt werden muss. Schließlich müsste der nach Kant und vielen, die ihm darin folgen, mit einem jeden Recht einhergehende Zwang moralisch begründet werden. Zugleich muss vermieden werden, dass der Zwang des öffentlichen Rechts gegenüber jenen, die nicht eingewilligt haben, diese als bloße Mittel zur öffentlichen Ordnung des Staates benutzen (Willaschek 2009 und 2012, 559). 
die Rechtsordnung der Staaten übernehmen. Der Würdegrundsatz umschreibt das Fundament wechselseitiger Achtung von Menschen als Rechtspersonen als eine quasi transzendentale Voraussetzung rechtsstaatlicher Ordnung. Alle Rechtspersonen im Staat haben Anspruch auf gleiche Achtung, d. h. sie haben Anspruch darauf, als Personen gleichermaßen respektiert zu werden. Als solche kommt ihnen unter anderem ein ,Recht, Rechte zu haben“ (so Hannah Arendts berühmte Formel, ${ }^{11} 2006$ [1955], 614) zu. Der Würdesatz soll dem Einzelnen in seinem irreduziblen Eigenwert einen schlechthin nicht antastbaren Freiheits- und Schutzbereich gegenüber Kollektivinteressen garantieren. Die primäre Struktur und Funktion des Würdeschutzes ist die einer deontologisch zu verstehenden Grenze dessen, was Rechtspersonen angetan werden darf. Diese Konzeption ist also keine auf einem (gegebenenfalls kommensurablen) Wert basierende Auffassung, sondern eine, die im Geiste der Zweck-an-sich und der Reich-der-Zwecke Formel des Kategorischen Imperativs dem Menschen einen absoluten Status zuerkennt. Basierend auf dem als transzendental angesehenen Grund der Würde eines jeden Menschen, nämlich der Autonomie der Person, wird der moralische und politische Sinn der Menschenrechte darin gesehen, einen Status von Personen als rechtlich, politisch und sozial Gleiche innerhalb ihrer normativen Ordnung zu etablieren (Rawls 1993, Kap. 3; O’Neill 1989).

Aufbauend auf dem moralisch basalen Status als Freie und Gleiche kann man sodann diskurstheoretisch-kantisch dafür argumentieren, dass Personen deshalb auch den bürgerlichen Status als gleichberechtigte normative Autorität bei der Konstruktion der politischen Ordnung zuerkannt bekommen müssen. Menschenwürde ist die „Würdigkeit eines jeden vernünftigen Subjects, ein gesetzgebendes Glied im Reiche der Zwecke zu sein“ (GMS, AA 04: 439). Ein solches gesetzgebendes Glied zu sein, heißt, nicht legitimatorisch übergangen zu werden und zu wissen, dass man andere nicht übergehen soll. Subjekte mit Menschenwürde sind deshalb Menschen mit einem unbedingt zu achtenden Recht auf Rechtfertigung (Forst 2007 und in diesem Band). So begründet der kantisch inspirierte gleiche Menschenwürdestatus als basalstes moralisches und juridisches Recht zugleich zum einen basale Schutzrechte für Individuen, zum anderen auch die gleichen Mitbestimmungsrechte bei der Konstruktion aller weiteren Grundrechte (vgl. Habermas 1992, Kap. 3). Ganz im kantischen Geiste wird so der Begriff der Menschenwürde als verbunden mit dem der Selbstbestimmung gesehen, in einer moralischen Bedeutung, die bereits eine politische Komponente enthält: Es geht um das Nichtbeherrschtwerden durch andere, nicht legitimierte Kräfte. Wir schulden uns und allen Personen Respekt vor ihrer Autonomie und damit die Achtung aller Personen als „Zwecke an sich selbst“. In dieser kantischen Konzeption wird also eine moralphilosophische Figur, nämlich Menschen als Zwecke an sich zu behandeln, in den politisch-staatlichen und transnationalen Bereich 
übertragen, auch wenn Kant das so - wie gezeigt - nicht vorgesehen hat. In einer gewissen kantischen Perspektive, nämlich der diskurstheoretischen Lesart des Kategorischen Imperativs, vermittelt das Recht auf Rechtfertigung zwischen der moralischen und der rechtlich-politischen Ebene. Ernst genommen wird damit Kants Insistieren, dass nur der omnilaterale Wille Rechte begründen kann. Dieser kantische Ansatz wird somit mehreren zentralen Aspekten des Kantischen Denkens gerecht, auch wenn er anders als Kant selbst verfährt.

b) Eine zweite naheliegende kantische Abweichung von der expliziten Theorie Kants besteht darin, eine republikanische Theorie globaler Ordnung zu konstruieren. So wie das Staatsrecht bei Kant die Bedingungen der Möglichkeit einer gerechten zivilen Ordnung in einem territorial begrenzten Staat ausbuchstabiert, so könnte man in Analogie eine gerechte internationale Ordnung entwerfen. Diese gerechte supra- oder transnationale Ordnung würde zwischen der freien Föderation republikanischer Staaten auf dem einen Ende und einem souveränen globalen republikanischen Weltstaat auf dem anderen Ende eines Spektrums von Möglichkeiten liegen. Indem man die Bedingungen der Möglichkeit einer solchen gerechten supra- oder transnationalen Ordnung ausbuchstabiert, käme man wahrscheinlich nahe an so etwas wie das gegenwärtige Verständnis der Menschenrechte. Bei dieser kantischen Theorievariante weicht man allerdings von Kant insofern ab, als man entgegen seinem Diktum des Nicht-Beherrschtwerdenkönnens Institutionen denken und dann schaffen muss, deren Aufgabe es unter anderem ist, in die innerstaatlichen Angelegenheiten von Republiken gegen deren Willen einzugreifen, zumindest wenn diese Menschenrechte auf ihrem Territorium nicht effektiv schützen, schützen können oder schützen wollen. Um das Interventionsrecht ansatzweise kantisch gegen Kant rechtfertigen zu können, muss die supra- oder transnationale Institution republikanisch verfasst sein, also den Gemeinwillen aller Völker und Individuen repräsentieren. Nur wenn diese zentrale Bedingung erfüllt ist, könnte es Kantianisch gerechtfertigt sein, dass man sich in gravierenden Fällen über das Urteil von Einzelstaaten zum Zwecke des Menschenrechtsschutzes hinwegsetzen darf. Für die heutige Menschenrechtsauffassung bedeutet das eine Veränderung zum status quo: Wenn immer eine Menschenrechtsverletzung ausgemacht wird, die das Eingreifen der Völkergemeinschaft verlangt, muss logisch die Existenz einer öffentlichen Repräsentation der ganzen Weltgemeinschaft unterstellt werden, die diesen Eingriff genehmigt. Damit bliebe man bei der Kantischen Idee, dass etwas nur dann ein Recht, hier ein Menschenrecht sein kann, wenn es öffentlich als dem Gemeinwillen entsprechend gerechtfertigt ist. Allerdings lässt sich das Einverständnis aller bei Kant keineswegs hypothetisch verstehen. Kant besteht in der Rechtslehre auf einer tatsächlichen offiziellen Repräsentation dessen, was die der Rechtsordnung Unterworfenen wirklich wollen. Man könne nicht wissen, was das 
Volk wolle, meint Kant, ohne eine tatsächliche Repräsentation dieses Volkswillens. Hypothetische Lösungen hingegen erlauben unterschiedliche Vorstellungen. Unterschiedliche Vorstellungen wiederum liefen letztlich auf zwei unterschiedliche Souveräne hinaus oder einen Konflikt zwischen dem Souverän und dem Volk. Das aber bedeutet für Kant - wie oben schon dargelegt - das gleiche wie die Aufgabe einer jeden rechtlich legitimen Ordnung überhaupt. Das dürfte vielleicht ein Grund sein, warum Kant selbst diesen republikanischen, demokratischen Weg zu einer transnationalen Weltordnung nicht gegangen ist. Wenn man ihn jedoch gehen will, bedeutet das, dass die heutigen Vereinten Nationen demokratisiert werden müssten, um menschenrechtliche Auflagen und Eingriffe in nur bedingt souveräne Staaten legitimieren zu können.

Zum Schluss: Für Kant sind alle Rechtsansprüche wesentlich politisch und nicht nur moralisch. Aus diesem Grund verlangen alle Rechte eine gesetzlich verfasste Republik als Schiedsrichter über konkurrierende Rechtsansprüche und Rechtsdurchsetzer. Das ist letztlich eine immanente Kritik des Naturrechts und seiner Tradition durch Kant. Naturrecht ist für Kant letztlich unvollständig und verlangt eine gesetzliche Grundlage, um unvermittelte moralische Ansprüche auf Naturrechte zu übertrumpfen. In dem Maße, in dem das heutige Menschenrechtsverständnis in einem seiner Elemente noch einen Kern der Naturrechtsauffassung in sich hat, ist es mit Kants eigener Theorie deshalb nicht vereinbar. In dem Maße, in dem kantische Menschenrechtsauffassungen sich als Ausdruck eines omnilateralen faktischen Gemeinwillens verstehen, können sie zu Recht den Titel „kantisch“ tragen.

\section{Literatur}

Arendt, Hannah ( ${ }^{11} 2006$ [1955]): Elemente und Ursprünge totaler Herrschaft. München: Pieper Verlag.

Bielefeld, Heiner (1998): Philosophie der Menschenrechte. Grundlagen eines weltweiten Freiheitsethos. Darmstadt: Wissenschaftliche Buchgesellschaft.

Byrd, Sharon/Hruschka, Joachim (2010): Kant's Doctrine of Right: A Commentary. Cambridge: Cambridge University Press.

Eberl, Oliver/Peter Niesen (2011): „Kommentar ,Zum Ewigen Frieden““. In: Immanuel Kant, Zum ewigen Frieden. Berlin: Suhrkamp Studienbibliothek, 89-416.

Flikschuh, Katrin (2008): „Reason, Right and Revolution: Kant and Locke“. In: Philosophy and Public Affairs 36, 375-404.

Flikschuh, Katrin (2015): „Human Rights in Kantian Mode: A Sketch“. In: Cruft, Rowan/ Liao, S. Matthew/Renzo, Massimo (Ed.): Philosophical Foundations of Human Rights. Oxford: Oxford University Press, 653-671.

Forst, Rainer (2007): Recht auf Rechtfertigung. Elemente einer konstruktivistischen Theorie der Gerechtigkeit. Frankfurt/M: Suhrkamp. 
Gosepath, Stefan (2009): „Sinn der Menschenrechte nach 1945“

In: Sandkühler, Hans-Jörg (Hg.): Menschenrechte in die Zukunft denken. 60 Jahre

Allgemeine Erklärung der Menschenrechte. Baden-Baden: Nomos, 35-47.

Guyer, Paul (2006): Kant. New York: Routledge.

Habermas, Jürgen (1992): Faktizität und Geltung. Frankfurt/M: Suhrkamp.

Habermas, Jürgen (1996): „Kants Idee des ewigen Friedens - aus dem historischen Abstand von 200 Jahren“. In: Ders., Die Einbeziehung des Anderen. Studien zur politischen Theorie. Frankfurt/M: Suhrkamp, 192-236

Höffe, Otfried (1990): Kategorische Rechtsprinzipien. Ein Kontrapunkt der Moderne. Frankfurt/M: Suhrkamp.

Höffe, Otfried (2001): „Königliche Völker“. Zu Kants kosmomolitischer Rechts- und Friedenstheorie. Frankfurt/M: Suhrkamp.

Höffe, Otfried (1999) (Hg.): Immanuel Kant. Metaphysische Anfangsgründe der Rechtslehre. Berlin: Akademie Verlag.

Höffe, Otfried (2011) (Hg.): Immanuel Kant. Zum ewigen Frieden. Berlin: Akademie Verlag.

Hoesch, Matthias (2015): „Weltbürgerrecht“. In: Willaschek, Marcus u. a. (Hg.): Kant-Lexikon. Berlin, New York: De Gruyter, 2620.

Horn, Christoph (2014): Nichtideale Normativität. Ein neuer Blick auf Kants politische Philosophie. Frankfurt/M: Suhrkamp.

Hruschka, Joachim (2015): „Menschenrecht“. In: Willaschek, Marcus u. a. (Hg.). Kant-Lexikon. Berlin, Boston: De Gruyter, 1518-9.

Kersting, Wolfgang (21993): Wohlgeordnete Freiheit. Immanuel Kants Rechts- und Staatsphilosophie. Frankfurt/M: Suhrkamp.

Klemme, Heiner F. (2012): „Immanuel Kant“. In: Pollmann, Arnd/Lohmann, Georg (Hg.): Menschenrechte. Ein interdisziplinäres Handbuch. Stuttgart: Metzler, 44-51.

König, Siegfried (1994): Zur Begründung der Menschenrechte: Hobbes-Locke-Kant, Freiburg/München: Verlag Karl Alber.

Lutz-Bachmann, Matthias/Bohman, James (1996) (Hg.): Frieden durch Recht. Kants Friedensidee und das Problem einer neuen Weltordnung. Frankfurt/M: Suhrkamp.

Mosayebi, Reza (2013): Das Minimum der reinen praktischen Vernunft. Vom Kategorischen Imperativ zum allgemeinen Rechtsprinzip bei Kant. Berlin, Boston: De Gruyter.

Müller, Jörg Paul (1999): „Das Weltbürgerrecht (§62) und Beschluss“. In: Höffe, Otfried (Hg.): Immanuel Kant. Metaphysische Anfangsgründe der Rechtslehre. Berlin: Akademie Verlag, 257-278.

O'Neill, Onora (1989): Constructions of Reason. Explorations of Kant's Practical Philosophy. Cambridge: Cambridge University Press.

Paton, Herbert James (1947): The Categorical Imperative. A Study in Kant's Moral Philosophy. London: Nachdruck Philadelphia: Penn University Press.

Rawls, John (1993): Political Liberalism. New York: Columbia University Press.

Ripstein, Arthur (2009): Force and Freedom. Kant's Legal and Political Philosophy. Cambridge (MA): Harvard University Press.

Rosen, Allen (1993): Kant's Theory of Justice. Ithaca, London: Cornell University Press.

Sangiovanni, Andrea (2015): „Why there Cannot be a Truly Kantian Theory of Human Rights“. In: Cruft, Rowan/Liao, S. Matthew/ Renzo, Massimo (Ed.): Philosophical Foundations of Human Rights. Oxford: Oxford University Press, 671-689. 
Schönecker, Dieter/Wood, Allen (2003): Immanuel Kant „Grundlegung zur Metaphysik der Sitten. “Ein einführender Kommentar. Paderborn: UTB.

Sensen, Oliver (2009): „Kant’s Conception of Human Dignity“. In: Kant-Studien 100, 309-331. Sensen, Oliver (2011): Kant on Human Dignity. Berlin, New York: De Gruyter.

Stilz, Anna (2009): Liberal Loyalty: Freedom, Obligation, and the State. Princeton, NJ: Princeton University Press.

Timmons, Mark (2000) (Ed.): Kant's Metaphysics of Morals: Interpretative Essays. Oxford: Oxford University Press.

Valentini, Laura (2012): „Human Rights, Freedom, and Political Authority“. In: Political Theory 40: $573-601$.

Willaschek, Marcus (2009): „Right and Coercion: Can Kant's Conception of Right Be Derived from His Moral Theory?“ In: International Journal of Philosophical Studies 17: 49-70.

Willaschek, Marcus (2012): „The Non-Derivability of Kantian Right from the Categorical Imperative: A Response to Nance“. In: International Journal of Philosophical Studies 20: 557-64. 